Article

\title{
Sc-Decorated Porous Graphene for High-Capacity Hydrogen Storage: First-Principles Calculations
}

\author{
Yuhong Chen ${ }^{1,2, *}$, Jing Wang ${ }^{1,2}$, Lihua Yuan ${ }^{2}$, Meiling Zhang ${ }^{2}$ and Cairong Zhang ${ }^{1,2}$ \\ 1 State Key Laboratory of Advanced Processing and Recycling of Non-Ferrous Metals, \\ Lanzhou University of Technology, Lanzhou 730050, China; mirror1217@163.com (J.W.); \\ zhcrxy@lut.cn (C.Z.) \\ 2 School of Science, Lanzhou University of Technology, Lanzhou 730050, China; yuanlh@lut.cn (L.Y.); \\ zhangml_2000@126.com (M.Z.) \\ * Correspondence: chenyh@lut.cn; Tel.: +86-931-297-3780
}

Received: 8 July 2017; Accepted: 31 July 2017; Published: 2 August 2017

\begin{abstract}
The generalized gradient approximation (GGA) function based on density functional theory is adopted to investigate the optimized geometrical structure, electron structure and hydrogen storage performance of Sc modified porous graphene (PG). It is found that the carbon ring center is the most stable adsorbed position for a single Sc atom on PG, and the maximum number of adsorbed $\mathrm{H}_{2}$ molecules is four with the average adsorption energy of $-0.429 \mathrm{eV} / \mathrm{H}_{2}$. By adding a second $\mathrm{Sc}$ atom on the other side of the system, the hydrogen storage capacity of the system can be improved effectively. Two Sc atoms located on opposite sides of the PG carbon ring center hole is the most suitable hydrogen storage structure, and the hydrogen storage capacity reach a maximum $9.09 \mathrm{wt} \%$ at the average adsorption energy of $-0.296 \mathrm{eV} / \mathrm{H}_{2}$. The adsorption of $\mathrm{H}_{2}$ molecules in the PG system is mainly attributed to orbital hybridization among $\mathrm{H}, \mathrm{Sc}$, and $\mathrm{C}$ atoms, and Coulomb attraction between negatively charged $\mathrm{H}_{2}$ molecules and positively charged $\mathrm{Sc}$ atoms.
\end{abstract}

Keywords: first principles; Sc modification; porous graphene; hydrogen storage

\section{Introduction}

Considerable research has been conducted to search for clean, renewable energy to meete growing energy demand [1]. Hydrogen has many advantages such as being recyclable, highly abundant, pollution-free, of high gravimetric energy density, and high combustion heat. Therefore hydrogen is considered to be an ideal carrier for energy storage [2]. However, the primary obstacle to hydrogen application is safe and efficient storage under ambient conditions [3,4]. To meet the criteria of the U.S. Department of Energy (DOE) and the International Energy Agency (IEA), the desirable hydrogen storage capacity of a promising material should be greater than $5.5 \mathrm{wt} \%$. The adsorption energy between $\mathrm{H}_{2}$ molecules and materials should be from 0.2 to $0.7 \mathrm{eV}$ [5]. Therefore, it is appropriate that hydrogen stores in solid materials in a molecular form [6].

Carbon nanostructures, such as graphene [7,8], fullerene [9,10], and nanotubes [11] have been investigated for solid-state hydrogen storage because of their high specific surface area, fast kinetics, and reversible hydrogen storage etc. However, $\mathrm{H}_{2}$ molecules bind weakly with pristine graphene, which is chemically too inert to act as a promising hydrogen storage material. Many studies have been performed to improve the chemical activity of graphene, such as doping or decoration by alkali metals [12,13], alkaline-earth metals [14-16], and transition metals [17-19]. The adsorption energy of hydrogen molecules on graphene can significantly be improved by the modification of transition metal (TM) atoms [20]. However, the large cohesive energy of TM atoms can easily lead to the formation of clusters on graphene. Research shows that the introduction of impurity atoms or vacancies can prevent the TM atoms from aggregating, thus increasing the hydrogen storage capacity of graphene [21]. 
Therefore, graphene which has a foraminiferous defect and doped atoms, decorated with TM atoms, has attracted attention.

Luo et al. [22] noted that the Sc-decorated single-vacancy defect graphene with three $\mathrm{N}$ atoms doped was the best medium for hydrogen storage, using Vienna Ab-initio Simulation Package (VASP) code within the GGA functional calculation, because each Sc atom could adsorb five $\mathrm{H}_{2}$ molecules with the adsorption energies from 0.2 to $0.4 \mathrm{eV}$. Using $\mathrm{DMol}^{3}$ code within the local density approximation (LDA) functional calculation, Omar Faye et al. [23] found that up to eight $\mathrm{H}_{2}$ molecules could be adsorbed by double-sided Pd-functionalized graphene. In addition, Omar Faye et al. [24] also studied hydrogen storage in $\mathrm{Cu}$-functionalized boron-doped graphene by the same method. A gravimetric hydrogen density of $4.231 \mathrm{wt} \%$ was reached when $\mathrm{H}_{2}$ adsorbed on double-sided $\mathrm{Cu}$-functionalized graphene. Using the $\mathrm{DMol}^{3}$ package within the LDA functional calculation, Y decorated B-doped graphene was studied as a potential carrier for hydrogen storage, in which the hydrogen storage capacity was 5.78 wt \%. The corresponding average adsorption energy was $-0.568 \mathrm{eV}$ per $\mathrm{H}_{2}$ molecule [25]. Although the average adsorption energy of $\mathrm{H}_{2}$ molecules is suitable for reversible hydrogen storage in these systems, the hydrogen storage capacity is close to the minimum $5.5 \mathrm{wt} \%$ of United States DOE.

Because of the good performance of graphene, other graphene-like materials have also attracted attention. Examples are silicene [26-28], monolayer black phosphorus [29], arsenene [30] and porous graphene [31]. Porous graphene (PG) was successfully fabricated by Bieri et al [32]. The inherent nanopores in porous graphene are well-developed and uniformly distributed, which portend highly effective hydrogen storage by a high specific surface area [33]. Du et al. [12] investigated Li-decorated PG by VASP code within the LDA, which found that the hydrogen storage capacity was up to $12 \mathrm{wt} \%$. However, the average binding energy of $0.243 \mathrm{eV}$ per $\mathrm{H}_{2}$ was overestimated due to the LDA function. Reunchan et al. [34] investigated the Ca-decorated PG and found that an isolated Ca atom could adsorb five hydrogen molecules with the average adsorption energy of $0.230 \mathrm{eV}$. Recently, Yuan et al. [35] studied Y-decorated porous graphene and they found that the maximum number of adsorbed hydrogen molecules around the $\mathrm{Y}$ atom was six and the average adsorption energy was $-0.297 \mathrm{eV}$.

Sc is the lightest TM atom, indicating that Sc-decorated PG could produce higher hydrogen storage capacity. In this paper, we calculate the most stable adsorption structure of Sc atoms on PG and the corresponding adsorption energy. In addition, the adsorption properties and adsorption mechanism of $\mathrm{H}_{2}$ molecules on the Sc decorated PG system are analyzed to better understand the effect of Sc atoms modified PG on the hydrogen storage properties.

\section{Calculation Details}

Our studies are performed using the plane wave CASTEP package [36] with the Perdew-Burke-Ernzerhof (PBE) of the generalized gradient approximation (GGA) exchange-correlation function [37]. Since the GGA function may underestimate the relatively weak adsorption energies, a pragmatic method to resolve this issue has been given by the dispersion-corrected density functional theory (DFT-D) approach. Calculations are performed using the DFT-D method to consider the van der Waals forces. All the atoms are relaxed in our calculations such that the force on each atom is less than $0.01 \mathrm{eV} / \AA$. The convergence tolerance energy is $5.0 \times 10^{-6} \mathrm{eV} /$ atom to realize the energy minimization. The self-consistent field (SCF) convergence threshold is $1.0 \times 10^{-6} \mathrm{eV} /$ atom. The calculation of the porous graphene unit cell satisfies the periodic boundary conditions. The vacuum thickness of $20 \AA$ is adopted to minimize the interlayer interaction. In order to ensure convergence and improve the accuracy of our calculations, the cutoff energy and the k-point are tested. Considering the test results and the computational cost, the cutoff energy is set to $500 \mathrm{eV}$ and the k-point is set to $7 \times 7 \times 1$.

The average adsorption energy of Sc atoms on PG layer is defined as:

$$
\bar{E}_{\mathrm{b}}=\left[E_{\mathrm{Sc}+\mathrm{PG}}-E_{\mathrm{PG}}-n E_{\mathrm{Sc}}\right] / n
$$


where $E_{\mathrm{Sc}+\mathrm{PG}}, E_{\mathrm{PG}}$ and $E_{\mathrm{Sc}}$ represent the total energy of the PG system with Sc atoms, the total energy of PG and the total energy of a free Sc atom, respectively. Moreover, $n$ represents the number of adsorbed Sc atoms.

The adsorption energy $\left(E_{\mathrm{ad}}\right)$ and average adsorption energy $\left(\bar{E}_{\mathrm{ad}}\right)$ of adsorbed $\mathrm{H}_{2}$ molecules are respectively defined as:

$$
\begin{gathered}
E_{\mathrm{ad}}=\left[E_{i \mathrm{H}_{2}+\mathrm{Sc}+\mathrm{PG}}-E_{(i-1) \mathrm{H}_{2}+\mathrm{Sc}+\mathrm{PG}}-E_{\mathrm{H}_{2}}\right] \\
\bar{E}_{\mathrm{ad}}=\left[E_{i \mathrm{H}_{2}+\mathrm{Sc}+\mathrm{PG}}-E_{\mathrm{Sc}+\mathrm{PG}}-i E_{\mathrm{H}_{2}}\right] / i
\end{gathered}
$$

where $E_{i \mathrm{H}_{2}+\mathrm{Sc}+\mathrm{PG}}$ and $E_{(i-1) \mathrm{H}_{2}+\mathrm{Sc}+\mathrm{PG}}$ are the total energy of the Sc-decorated PG system with $i$ and $(i-1) \mathrm{H}_{2}$ molecules adsorbed, respectively. $E_{\mathrm{H}_{2}}$ represents the energy of an isolated $\mathrm{H}_{2}$ molecule.

The fully optimized structure of a porous graphene unit cell is shown in Figure 1. The calculated lattice constant is $7.49 \AA$, which agrees well with the experimental value of $7.40 \AA$ [32]. There are two kinds of $C$ atoms on PG. $C 1$ is connected with three $C$ atoms, while $C 2$ is connected with two $C$ atoms and one $\mathrm{H}$ atom. The bond length of $\mathrm{C} 1-\mathrm{C} 1$ is $1.489 \AA, \mathrm{C} 1-\mathrm{C} 2$ is $1.399 \AA, \mathrm{C} 2-\mathrm{H}$ is $1.085 \AA$. Our results are very close to the values of Brunetto [38], who gets the results using the $\mathrm{DMol}^{3}$ package with the GGA-BLYP function. In addition, $\mathrm{Li}$ [39] calculates the bond length of C2-H at $1.080 \AA$ using the $\mathrm{DMol}^{3}$ package with the GGA-PW91 function. The result is consistent with our value of $1.085 \AA$. The direct band gap is calculated to be $2.399 \mathrm{eV}$ using the GGA with the PBE function, which coincides well with the results of Rao [37] who obtains the value of band gap at $2.400 \mathrm{eV}$ based on VASP code with the PBE of the GGA function. The above test results show that the calculation method and parameters used in this paper are reliable.

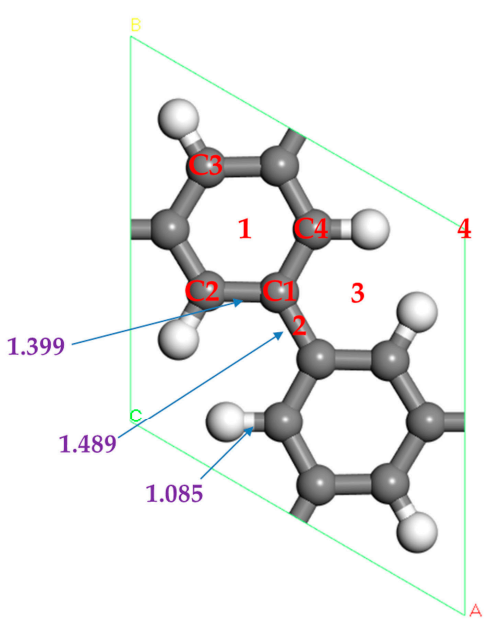

Figure 1. The optimized atomic structure of porous graphene. The gray and white balls represent $\mathrm{C}$ and $\mathrm{H}$ respectively.

\section{Results and Discussion}

\subsection{Single Sc Atom Decorated PG}

\subsubsection{The Adsorption Structure of Single Sc Atom Decorated PG}

Now we study the adsorption of one Sc atom on PG. There are four different symmetric positions for a Sc atom adsorption, which are shown in Figure 1 and named 1, 2, 3, 4, indicating the hollow center of $\mathrm{C}$ ring, the bridge of $\mathrm{C}-\mathrm{C}$ bond, the hollow center of a half $\mathrm{C}$ ring, and a large hexagonal pore, respectively. It is found that the 1 position is the favorite adsorption site for a Sc atom on PG, and this position is the same as the adsorption of a Sc atom on graphene [22]. The favorite configuration after full relaxation is shown in Figure 2a. We find that the Sc atom is a little deviation from the hollow center of the C-hexagon, and the adsorption energy is $-2.143 \mathrm{eV}$ for a Sc atom on PG. 


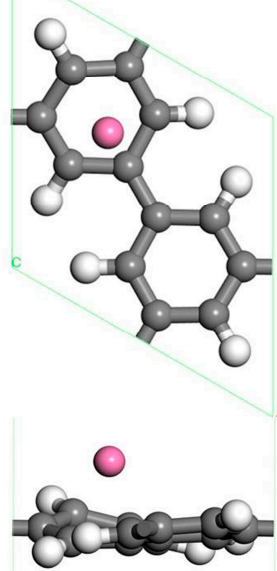

(a)

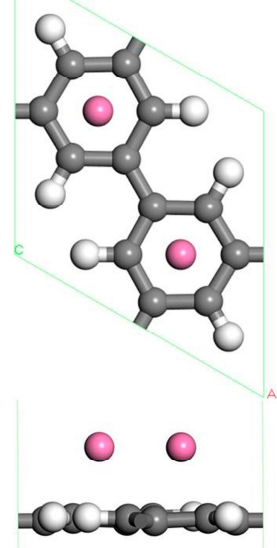

(b)

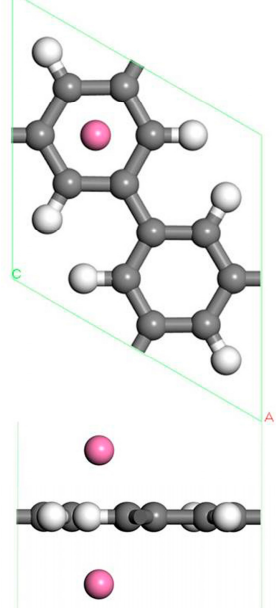

(c)

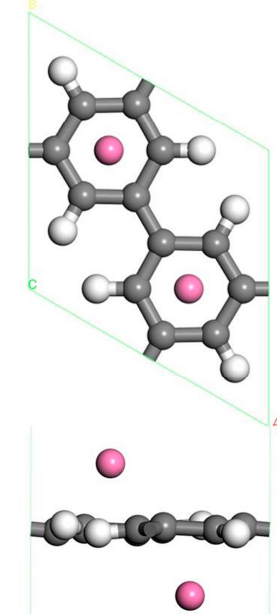

(d)

Figure 2. The optimized atomic structure of Sc atom decorated PG. (a) single Sc atom decorated PG; (b) two Sc atoms decorated single-sided PG; (c) two Sc atoms decorated double-sided PG at the same hole; (d) two Sc atoms decorated double-sided PG at the adjacent hole. The gray, white and pink balls in this and following figures denote $\mathrm{C}, \mathrm{H}$ and Sc atoms, respectively.

We also calculate the adsorption of Sc on perfect graphene to further investigate the effect of graphene defects on Sc adsorption. The adsorption energy of the Sc atom on the intact graphene is $-1.499 \mathrm{eV}$, which is calculated using the CASTEP package within the PBE of the GGA function. This is very close to the value of $-1.550 \mathrm{eV}$, which is calculated based on VASP code with the PBE of the GGA function [22]. The adsorption energy of Sc atoms on perfect graphene is far less than that on PG. After adsorption, the positive charges of the Sc atom on the perfect graphene and PG are $1.24 \mathrm{e}, 1.33 \mathrm{e}$, respectively. In other words, more charge transfers in the Sc-decorated PG system, and this stems from the fact that the interaction between Sc atoms and PG is stronger than the interaction between Sc atoms and perfect graphene. From the partial densities of states (PDOS) of Sc and C in Figure 3, we can learn the peak of the $C 2 p$ orbital overlap with the peak of the $S c 3 d$ orbital at $-2.50 \mathrm{eV}$, and this suggests a strong hybridization between $\mathrm{C}$ and Sc atoms.

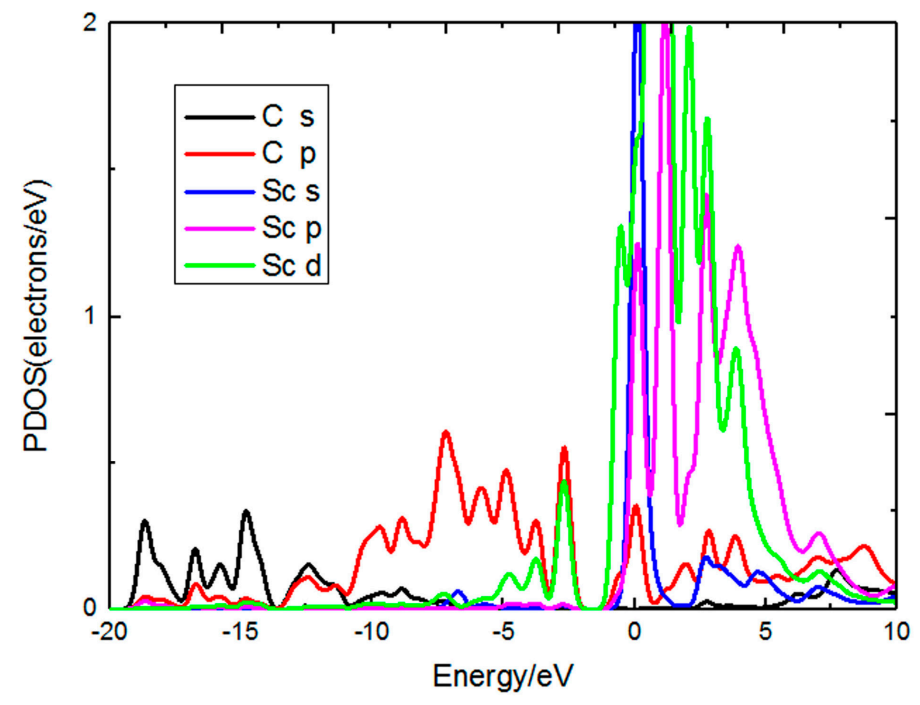

Figure 3. PDOS of Sc-decorated PG system. 


\subsubsection{The Adsorption of $\mathrm{H}_{2}$ Molecules on Single Sc-Decorated PG}

The optimized geometries for $\mathrm{H}_{2}$ molecules adsorbed on the Sc-decorated porous graphene are shown in Figure 4, and Table 1 lists the adsorption energy and the average adsorption energy of $\mathrm{H}_{2}$ molecules. There are several positions for single $\mathrm{H}_{2}$ molecule adsorption, including the bridge of the $\mathrm{C}-\mathrm{C}$ bond, the bridge of the $\mathrm{C}-\mathrm{H}$ bond, the top of the $\mathrm{C}$ atom and the top of the Sc atom. It is found that the favorite position for the first $\mathrm{H}_{2}$ molecule is above the $\mathrm{C}-\mathrm{H}$ bond. The binding energy is $-0.401 \mathrm{eV}$, and the bond length is elongated to $0.818 \AA$. In order to study the hydrogen storage capacity of the system, we add other $\mathrm{H}_{2}$ molecules to the system one by one. From Table 1, we can see that the adsorption energy of $\mathrm{H}_{2}$ molecules first increases and then decreases for the consecutive adsorption of the $\mathrm{H}_{2}$ molecule. However, $d_{\mathrm{Sc}-\mathrm{C}}$ (the nearest distance between the $\mathrm{Sc}$ atom and the $\mathrm{C}$ atom of porous graphene) is the opposite, which means that the adsorption of $\mathrm{H}_{2}$ molecules influences the binding between the $\mathrm{Sc}$ atom and porous graphene. It is very interesting to note that almost all $\mathrm{H}_{2}$ molecules are symmetrically distributed around the $\mathrm{Sc}$ atom because of the symmetry of the bonding configuration of $\mathrm{H}_{2}$ molecules. In addition, the first four $\mathrm{H}_{2}$ molecules are parallel to the PG sheet and stay on the same plane. When the fifth $\mathrm{H}_{2}$ molecule is added to the system, it moves to an upper layer after relaxation, as shown in Figure 4e. This effect may be due to the limited space around the Sc atom and the repulsive interaction between the adsorbed $\mathrm{H}_{2}$ molecules [40]. Therefore, the $\mathrm{H}-\mathrm{H}$ bond length of the fifth $\mathrm{H}_{2}$ molecule is $0.756 \AA$ and this $\mathrm{H}_{2}$ molecule has the smallest adsorption energy of $-0.093 \mathrm{eV}$, which is weak for hydrogen storage application [41]. The system of the single Sc atom decorated porous graphene can adsorb a maximum of $4 \mathrm{H}_{2}$ molecules because of the two-layer arrangement. Therefore, the hydrogen storage capacity is $3.94 \mathrm{wt} \%$ and the average adsorption energy is $-0.429 \mathrm{eV} / \mathrm{H}_{2}$, which is higher than that of Sc modified defect graphene with three $\mathrm{N}$ atoms doped [22].

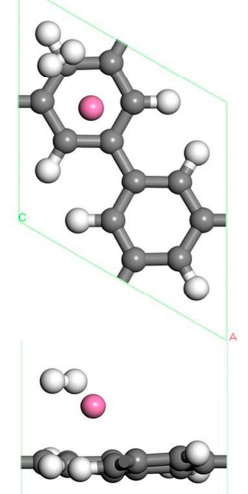

(a)

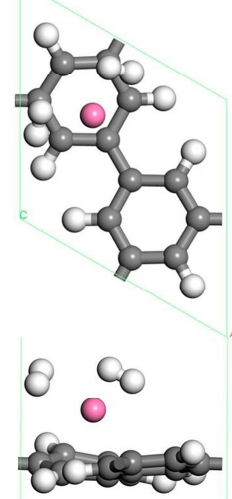

(b)

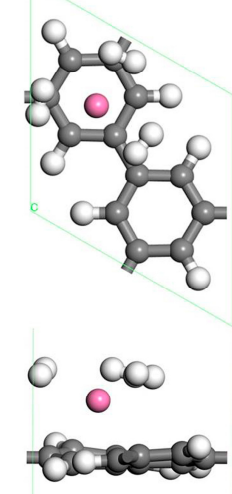

(c)

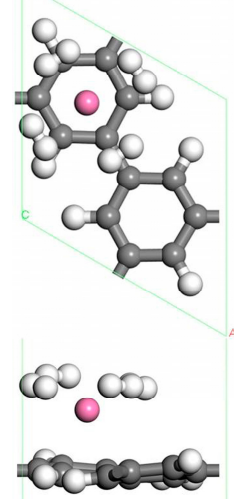

(d)

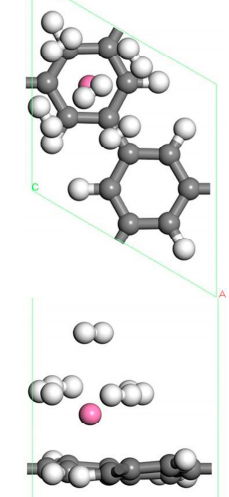

(e)

Figure 4. The optimized atomic structures of the Sc atom decorated PG with (a) one $\mathrm{H}_{2}$ molecule; (b) two $\mathrm{H}_{2}$ molecules; (c) three $\mathrm{H}_{2}$ molecules; (d) four $\mathrm{H}_{2}$ molecules; (e) five $\mathrm{H}_{2}$ molecules adsorbed.

Table 1. The adsorption energy, average adsorption energy and the nearest distance between Sc and $\mathrm{C}$ of porous graphene for $\mathrm{H}_{2}$ adsorbed on single Sc decorated PG.

\begin{tabular}{cccc}
\hline Number of $\mathbf{H}_{\mathbf{2}}$ & $\mathbf{E}_{\mathbf{a d}}(\mathbf{e V})$ & $\overline{\mathbf{E}}_{\mathbf{a d}}(\mathbf{e V})$ & $\boldsymbol{d}_{\mathbf{S c}-\mathbf{C}}(\AA)$ \\
\hline $1 \mathrm{H}_{2}$ & -0.401 & -0.401 & 2.348 \\
$2 \mathrm{H}_{2}$ & -0.718 & -0.559 & 2.217 \\
$3 \mathrm{H}_{2}$ & -0.422 & -0.514 & 2.291 \\
$4 \mathrm{H}_{2}$ & -0.174 & -0.429 & 2.451 \\
$5 \mathrm{H}_{2}$ & -0.093 & -0.361 & 2.477 \\
\hline
\end{tabular}

In order to investigate the interaction between the $\mathrm{Sc}$ atom and adsorbed $\mathrm{H}_{2}$ molecules, the partial densities of states for the $\mathrm{H}_{2}$ molecules and the Sc atom are plotted in Figure 5. It can be observed that 
there is a band broadening around $-9 \mathrm{eV}$, which indicates an $\mathrm{H}_{2}-\mathrm{H}_{2}$ interaction. In addition, there is a strong hybridization between $\mathrm{H}_{2}$ 1s orbitals and $\mathrm{Sc}_{\mathrm{c}} 3 \mathrm{~d}$ orbitals around -10.5 to $-8.0 \mathrm{eV}$, since the orbital overlap is between $1 \mathrm{~s}$ of $\mathrm{H}_{2}$ and $3 \mathrm{~d}$ of $\mathrm{Sc}$. In the range -1.5 to $0 \mathrm{eV}$, the adsorption of $1 \sim 4 \mathrm{H}_{2}$ molecules can be seen to have overlapping peaks between $\mathrm{H}_{2}$ 1s orbital and Sc $3 \mathrm{~d}$ orbital, suggesting that in this interval $\mathrm{H}_{2}$ molecules and the Sc atom also exist in hybridization, which may be one of the reasons why the first four $\mathrm{H}_{2}$ molecules have a strong interaction with the Sc atom. From the PDOS of $\mathrm{H}_{2}$ molecules, we can see that the 1 s orbital mainly distributes in $\sigma$ bonding state, namely a bonding state dominates the 1 s orbital and there is no $\mathrm{H}_{2}$ molecular dissociation.

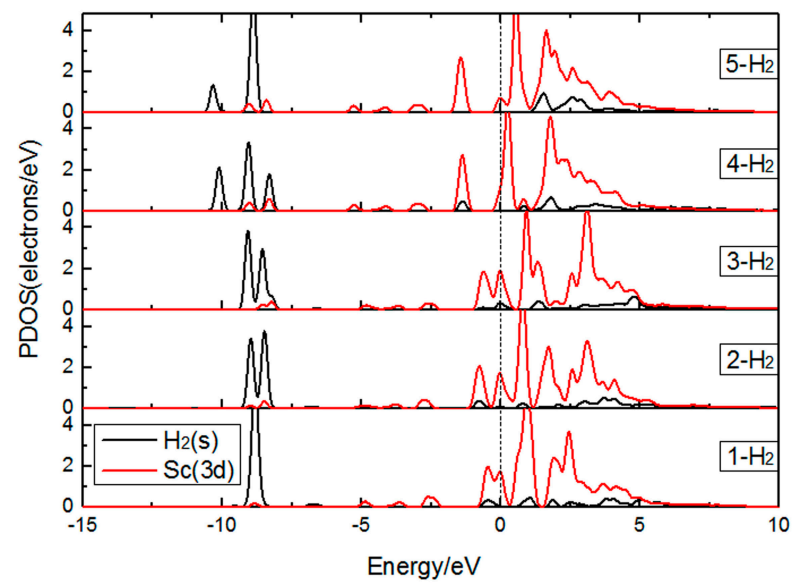

Figure 5. PDOS of Sc-decorated PG with one to five $\mathrm{H}_{2}$ molecules adsorbed.

Given the Mulliken charge population before and after $\mathrm{H}_{2}$ molecules adsorption, the bond strength and charge transfer between atoms could be analyzed. The Mulliken population analysis of Sc-PG system with and without one $\mathrm{H}_{2}$ molecule adsorbed are shown in Table 2. $\mathrm{H} 1$ and $\mathrm{H} 2$ represent the two hydrogen atoms of the adsorbed hydrogen molecule, and C2, C3 and C4 are the three C atoms that charge transfer most in the PG layer (as shown in Figure 1). After the first $\mathrm{H}_{2}$ adsorption, the charge of each atom of the $\mathrm{H}_{2}$ molecule is $-0.13 \mathrm{e}$, while the Sc atom carries the positive charge of 1.56 e. According to Figure 5, Sc 3d orbital and the $\mathrm{H}_{2}$ molecule of bonding and antibonding state are hybridizations. Therefore, $\mathrm{H}_{2}$ molecule transfers charge to the $\mathrm{Sc}$ atom, and then Sc atom back donates charge to the antibonding orbital of the $\mathrm{H}_{2}$ molecule. As a result, the $\mathrm{H}_{2}$ molecule carries a more negative charge. There is Coulomb attraction between the negatively charged $\mathrm{H}_{2}$ molecule and positively charged Sc atom. This is consistent with the case of Ca-decorated graphene for hydrogen storage [42]. The Coulomb interaction enhances the adsorption of the $\mathrm{H}_{2}$ molecule. Although the bond length of the $\mathrm{H}_{2}$ molecule is stretched, the $\mathrm{H}_{2}$ molecule remains its molecular bond. After one $\mathrm{H}_{2}$ molecule adsorption, the negative charge of $\mathrm{C} 2$ and $\mathrm{C} 4$ atoms increase. However, the negative charge of $\mathrm{C} 3$ nearest the $\mathrm{H}_{2}$ molecule is reduced by about $0.15 \mathrm{e}$, which indicates that these $\mathrm{C}$ atoms also play a role in $\mathrm{H}_{2}$ molecular adsorption.

Table 2. Mulliken population analysis of the Sc-PG system before and after one $\mathrm{H}_{2}$ molecule adsorption.

\begin{tabular}{ccccccccc}
\hline \multirow{2}{*}{ Atom } & \multicolumn{3}{c}{ Before Adsorption (e) } & \multicolumn{6}{c}{ After Adsorption (e) } \\
\cline { 2 - 9 } & $\mathbf{s}$ & $\mathbf{p}$ & $\mathbf{d}$ & Charge & $\mathbf{s}$ & $\mathbf{p}$ & $\mathbf{d}$ & Charge \\
\hline H1 & 1.00 & - & - & - & 1.13 & - & - & -0.13 \\
H2 & 1.00 & - & - & - & 1.13 & - & - & -0.13 \\
C2 & 1.19 & 3.19 & - & -0.38 & 1.19 & 3.23 & - & -0.42 \\
C3 & 1.20 & 3.28 & - & -0.48 & 1.18 & 3.15 & - & -0.33 \\
C4 & 1.19 & 3.20 & - & -0.39 & 1.19 & 3.23 & - & -0.42 \\
Sc & 0.18 & 5.84 & 1.65 & 1.33 & 0.04 & 5.63 & 1.77 & 1.56 \\
\hline
\end{tabular}


The charge transfer of the Sc-PG system can be intuitively observed through charge density difference. Therefore, the charge density difference $\Delta \rho$ is defined as:

$$
\Delta \rho=\rho_{i \mathrm{H}_{2}+\mathrm{Sc}+\mathrm{PG}}-\rho_{i \mathrm{H}_{2}}-\rho_{\mathrm{Sc}+\mathrm{PG}}
$$

where $\rho_{i \mathrm{H}_{2}+\mathrm{Sc}+\mathrm{PG}}$ is the electron charge density of the total system, $\rho_{i \mathrm{H}_{2}}$ is the charge density of $i$ adsorbed $\mathrm{H}_{2}$ molecules, and $\rho_{\mathrm{Sc}+\mathrm{PG}}$ is the charge density of the Sc decorated PG system. The charge density difference for the system Sc-PG with $\mathrm{H}_{2}$ molecules adsorbed is shown in Figure 6. The blue and yellow isosurface represents space charge accumulation and depletion, and the value for the isosurface is $0.007 \mathrm{e} / \AA^{3}$. It is easy to see that the charge transfer is mainly distributed in regions between $\mathrm{H}_{2}$ molecules and the Sc atom, indicating a strong interaction between them. The charge transfer of $\mathrm{C}$ atoms in $\mathrm{PG}$ is also observed, which indicates that these $\mathrm{C}$ atoms also play a role in $\mathrm{H}_{2}$ molecular adsorption. It demonstrates the $\mathrm{H}_{2}$ molecules are polarized such that charge depletion and accumulation at both ends of $\mathrm{H}_{2}$ molecules can be observed in Figure 6b. Combining the Mulliken charge population, each $\mathrm{H}$ atom of $\mathrm{H}_{2}$ molecules carries a negative charge of $-0.16 \mathrm{e}$ and $-0.14 \mathrm{e}$, and it has a Coulomb attraction with a positively charged Sc atom after polarization. Consequently, the adsorption of hydrogen molecules in the Sc-decorated PG system is due to the orbital hybridization among H, Sc, C atoms, and Coulomb attraction between negatively charged $\mathrm{H}_{2}$ molecules and positively charged Sc atoms.

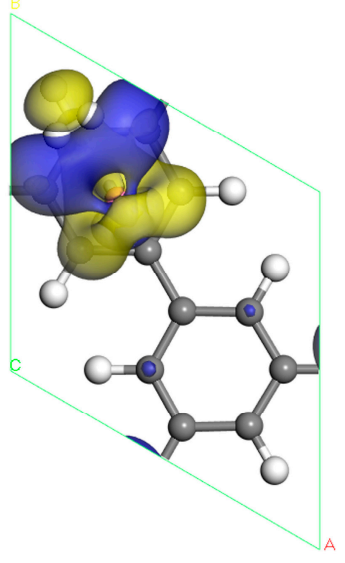

(a)

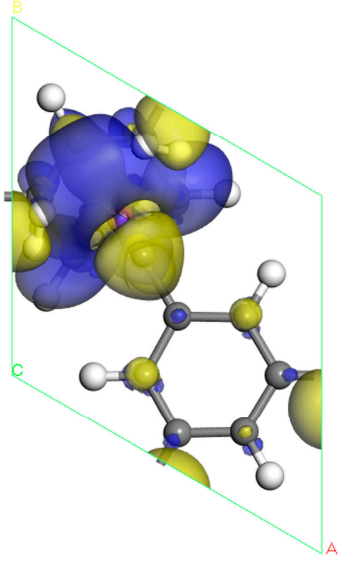

(b)

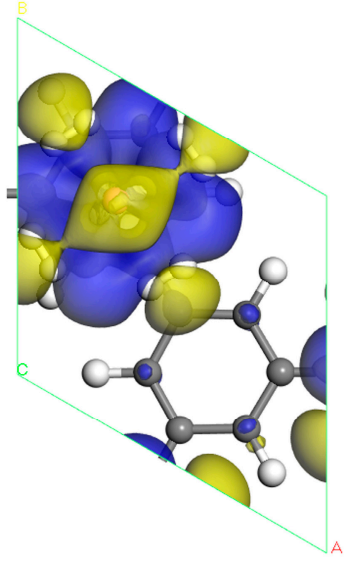

(c)

Figure 6. Electronic charge density difference for Sc-decorated PG system in the presence of (a) one $\mathrm{H}_{2}$ molecule; (b) two $\mathrm{H}_{2}$ molecules; (c) four $\mathrm{H}_{2}$ molecules.

\subsection{Two Sc Atoms Decorated PG}

\subsubsection{The Adsorption Structure of Two Sc Atoms Decorated PG}

The cohesive energy of Sc atoms is $3.90 \mathrm{eV}$ [43], which is much bigger than the binding energy of Sc atoms on PG. In order to test the possibility of Sc aggregation, we add the second Sc atom in the system on the same side. Figure $2 \mathrm{~b}$ presents the most stable optimized geometry for two Sc atoms adsorbed on PG. We find that the second Sc atom is more likely to adsorb on the center of another C hexagon rather than aggregate with the first $\mathrm{Sc}$ atom, and this is mainly because the Coulomb repulsion between two Sc atoms (both Sc atoms carry $0.87 \mathrm{eV}$ positive charge) and the strong interaction between Sc atoms and PG. The average binding energy of two Sc atoms on PG is $-3.195 \mathrm{eV}$, which implies that the interaction between the second Sc atom and PG is stronger than the first. In addition, the large distance of $4.322 \AA$ between two Sc atoms may be another reason for resistance clusters.

To examine further the space for hydrogen storage, this paper also studies two Sc atoms decorated double-sided PG. Each transition metal atom is an active adsorption point, and transition metal atoms modified double-sided PG can increase the hydrogen storage area. Based on the Figure 2a system, 
there are four adsorption positions for the second Sc atom on the opposite side of PG. The stable structures after relaxation are shown in Figure $2 \mathrm{c}, \mathrm{d}$ and the average adsorption energies of the Sc atom are $-2.813 \mathrm{eV}$ and $-2.537 \mathrm{eV}$. The hydrogen adsorption energy and storage properties of these two models are investigated in our work since the binding energy of Sc atoms has little difference.

\subsubsection{The Hydrogen Storage Capacity of Two Sc Atoms Modified PG System}

As shown in Figure $7 \mathrm{a}$, about ten $\mathrm{H}_{2}$ molecules can be adsorbed in the two Sc single-sided modified PG system without aggregation. The average adsorption energy is about $-0.268 \mathrm{eV} / \mathrm{H}_{2}$, and the hydrogen storage capacity is $7.69 \mathrm{wt} \%$. The cohesive energy of the Sc atom is higher than its binding energy on $\mathrm{PG}$, and after $\mathrm{H}_{2}$ adsorption the two $\mathrm{Sc}$ atoms are slightly closer to each other. This means that $\mathrm{Sc}$ aggregation may occur on the porous graphene, but from the optimized structure we not find Sc atom bonding with each other although Sc atoms can move freely. The average binding energy of two Sc atoms on PG is $-3.195 \mathrm{eV}$, which means that the adsorption structure of the two Sc atoms on the PG is more stable than that of a single Sc on the PG. This indicates adsorption structures are stable. After testing, we found that low concentrations of Sc atoms do not aggregate on PG. This phenomenon may be due to the Coulomb repulsion between two Sc atoms and the strong interaction between Sc atoms and PG, as well as the pore structure of the porous graphene. Given that the hydrogen storage space of PG on a single side is limited, to further improve the hydrogen storage capacity, two Sc atoms modified double-sided PG are also considered, as shown in Figure 2c,d. After hydrogen storage, the optimized structures are shown in Figure $7 \mathrm{~b}$,c. And the distance between the $\mathrm{Sc}$ atoms adsorbed on both sides of PG is large enough to prevent the aggregation. The Figure $7 \mathrm{~b}$ system can adsorb ten $\mathrm{H}_{2}$ molecules, the average adsorption energy of $\mathrm{H}_{2}$ molecules is $-0.292 \mathrm{eV}$, and the hydrogen storage capacity is $7.69 \mathrm{wt} \%$. The system of Figure 7c contains twelve $\mathrm{H}_{2}$ molecules adsorbed on Sc-decorated both sides of PG. The average adsorption energy of hydrogen in this system is $-0.296 \mathrm{eV} / \mathrm{H}_{2}$ higher than that of $-0.23 \mathrm{eV} / \mathrm{H}_{2}$ in the Y-PG system, and the hydrogen storage capacity is up to $9.09 \mathrm{wt} \%$, higher than that of $7.87 \mathrm{wt} \%$ in the Y-PG system [35].

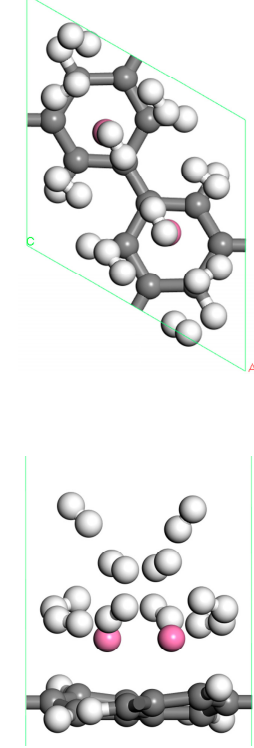

(a)
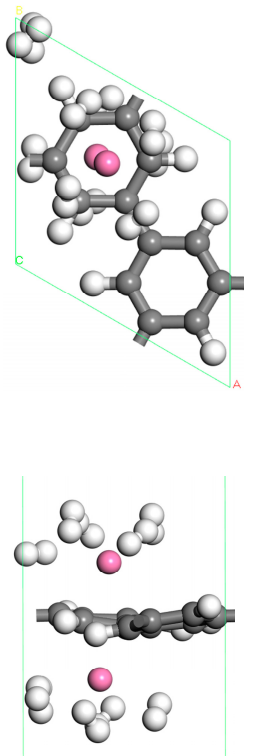

(b)

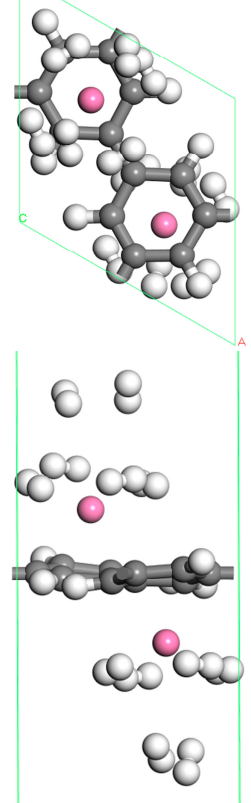

(c)

Figure 7. The optimized geometries for $\mathrm{H}_{2}$ molecules adsorbed on Sc-decorated PG. (a) two Sc atoms decorated single-sided PG; (b) two Sc atoms decorated double-sided PG at the same hole; (c) two Sc atoms decorated double-sided PG at the adjacent hole. 
The ideal adsorption energy between $\mathrm{H}_{2}$ molecules and materials should remain in the range of $0.2 \sim 0.7 \mathrm{eV} / \mathrm{H}_{2}$, which is between the physical and chemical adsorption state. It will be beneficial to hydrogen storage and release under the environmental temperature and pressure. In our work, the average adsorption energies of $\mathrm{H}_{2}$ molecules adsorbed on graphene are from -0.060 to $-0.075 \mathrm{eV} / \mathrm{H}_{2}$. The adsorption energy is in the range of $-0.061 \sim-0.104 \mathrm{eV} / \mathrm{H}_{2}$ for $\mathrm{H}_{2}$ molecules adsorbed on PG. The adsorption energy of $\mathrm{H}_{2}$ molecules at the 4 sites in Figure 1 (in the same plane with $\mathrm{PG}$ ) is positive and is the most unstable adsorption site. Therefore, the adsorption energy of $\mathrm{H}_{2}$ molecules adsorb on intrinsic graphene and PG is very small, which is not conducive to hydrogen storage.

The three systems of two Sc atoms modified PG for hydrogen storage are shown in Figure 7a-c. Their average adsorption energies are $-0.268 \mathrm{eV} / \mathrm{H}_{2},-0.292 \mathrm{eV} / \mathrm{H}_{2},-0.296 \mathrm{eV} / \mathrm{H}_{2}$ respectively, which is small in the ideal range. The systems of Figure 7a,b have the same hydrogen storage capacity, but the average adsorption energy of Figure $7 \mathrm{~b}$ is bigger than that of Figure $7 \mathrm{a}$. The hydrogen storage capacity of Figure $7 \mathrm{c}$ is the largest of the three systems, and the average adsorption energy of $\mathrm{H}_{2}$ molecules is the largest. As a result, the system of Figure $7 \mathrm{c}$ is the best structure for reversible hydrogen storage.

In addition, in comparing these systems (Figures $4 \mathrm{e}$ and $7 \mathrm{a}, \mathrm{c}$ ) we find an interesting phenomenon: The $\mathrm{H}_{2}$ molecules in Figure 7a are distributed in three layers, so the average adsorption energy is small $\left(-0.268 \mathrm{eV} / \mathrm{H}_{2}\right)$. In Figures $4 \mathrm{e}$ and $7 \mathrm{c}$, there are two layers of $\mathrm{H}_{2}$ molecules, and the average adsorption energies of $-0.429,-0.296 \mathrm{eV} / \mathrm{H}_{2}$ are larger. To sum up, two Sc atoms located above the adjacent $\mathrm{C}$ ring in double-sided $\mathrm{PG}$ is the most suitable structure for hydrogen storage. Therefore, Sc-decorated PG is a promising material for hydrogen storage.

\section{Conclusions}

In summary, based on the density functional theory, we studied the hydrogen storage properties of the Sc-PG system. For a single Sc atom, the most stable adsorption position on PG is the carbon hexagon center with an adsorption energy of $-2.143 \mathrm{eV}$. Four $\mathrm{H}_{2}$ molecules can be adsorbed around an Sc atom with an average adsorption energy of $-0.429 \mathrm{eV} / \mathrm{H}_{2}$. Analyzing the electronic structure of the system, the adsorption of $\mathrm{H}_{2}$ molecules in the PG system is mainly attributed to orbital hybridization among $\mathrm{H}, \mathrm{Sc}, \mathrm{C}$ atoms. The largest hydrogen storage capacity structure is two Sc atoms located above the adjacent $\mathrm{C}$ ring in a double side of PG. The system can absorb twelve $\mathrm{H}_{2}$ molecules with an average adsorption energy of $-0.296 \mathrm{eV} / \mathrm{H}_{2}$, and the hydrogen storage capacity is $9.09 \mathrm{wt} \%$. Therefore, Sc-decorated porous graphene may become one of the most promising hydrogen storage materials.

Acknowledgments: This work is supported by the National Natural Science Foundation of China (No. 51562022) and the Special Program for Applied Research on Super Computation of the NSFC-Guangdong Joint Fund (the second phase).

Author Contributions: Yuhong Chen designed the project, Jing Wang and Lihua Yuan performed the calculations, Yuhong Chen and Jing Wang prepared the manuscript, Cairong Zhang revised the paper, Lihua Yuan and Meiling Zhang analyzed the data, and all authors discussed the results and commented on the manuscript.

Conflicts of Interest: The authors declare no conflict of interest.

\section{References}

1. Masika, E.; Mokaya, R. Exceptional gravimetric and volumetric hydrogen storage for densified zeolite templated carbons with high mechanical stability. Energy Environ. Sci. 2014, 7, 427-434. [CrossRef]

2. Wang, Y.; Ji, Y.; Li, M.; Yuan, P.; Sun, Q.; Jia, Y. Li and Ca Co-decorated carbon nitride nanostructures as high-capacity hydrogen storage media. J. Appl. Phys. 2011, 110, 94311. [CrossRef]

3. Dillon, A.C.; Jones, K.M.; Bekkedahl, T.A.; Kiang, C.H.; Bethune, D.S.; Heben, M.J. Storage of hydrogen in single-walled carbon nanotubes. Nature 1997, 386, 377-379. [CrossRef]

4. Steele, B.C.; Heinzel, A. Materials for fuel-cell technologies. Nature 2001, 414, 345-352. [CrossRef] [PubMed]

5. Zhang, Z.W.; Li, J.C.; Jiang, Q. Hydrogen Adsorption on Eu/SWCNT Systems: A DFT Study. J. Phys. Chem. C 2010, 114, 7733-7737. [CrossRef] 
6. Sahaym, U.; Norton, M.G. Advances in the application of nanotechnology in enabling a 'hydrogen economy'. J. Mater. Sci. 2008, 43, 5395-5429. [CrossRef]

7. Ataca, C.; Aktürk, E.; Ciraci, S.; Ustunel, H. High-capacity hydrogen storage by metallized graphene. Appl. Phys. Lett. 2008, 93, 43123. [CrossRef]

8. Kim, G.; Jhi, S.-H.; Lim, S.; Park, N. Crossover between multipole Coulomb and Kubas interactions in hydrogen adsorption on metal-graphene complexes. Phys. Rev. B 2009, 79. [CrossRef]

9. Chandrakumar, K.R.S.; Ghosh, S.K. Alkali-Metal-Induced Enhancement of Hydrogen Adsorption in $\mathrm{C}_{60}$ Fullerene: An ab Initio Study. Nano Lett. 2008, 8, 13-19. [CrossRef] [PubMed]

10. Sun, Q.; Wang, Q.; Jena, P. Functionalized heterofullerenes for hydrogen storage. Appl. Phys. Lett. 2009, 94, 13111. [CrossRef]

11. Seenithurai, S.; Pandyan, R.K.; Kumar, S.V.; Saranya, C.; Mahendran, M. Al-decorated carbon nanotube as the molecular hydrogen storage medium. Int. J. Hydrogen Energy 2014, 39, 11990-11998. [CrossRef]

12. Du, A.; Zhu, Z.; Smith, S.C. Multifunctional Porous Graphene for Nanoelectronics and Hydrogen Storage: New Properties Revealed by First Principle Calculations. J. Am. Chem. Soc. 2010, 132, 2876-2877. [CrossRef] [PubMed]

13. Wang, Y.; Meng, Z.; Liu, Y.; You, D.; Wu, K.; Lv, J.; Wang, X.; Deng, K.; Rao, D.; Lu, R. Lithium decoration of three dimensional boron-doped graphene frameworks for high-capacity hydrogen storage. Appl. Phys. Lett. 2015, 106, 63901. [CrossRef]

14. Hussain, T.; Pathak, B.; Ramzan, M.; Maark, T.A.; Ahuja, R. Calcium doped graphane as a hydrogen storage material. Appl. Phys. Lett. 2012, 100, 183902. [CrossRef]

15. Song, N.; Wang, Y.; Zheng, Y.; Zhang, J.; Xu, B.; Sun, Q.; Jia, Y. New template for Li and Ca decoration and hydrogen adsorption on graphene-like SiC: A first-principles study. Comput. Mater. Sci. 2015, 99, 150-155. [CrossRef]

16. Gao, Y.; Zhao, N.; Li, J.; Liu, E.; He, C.; Shi, C. Hydrogen spillover storage on Ca-decorated graphene. Int. J. Hydrogen Energy 2012, 37, 11835-11841. [CrossRef]

17. Lebon, A.; Carrete, J.; Gallego, L.J.; Vega, A. Ti-decorated zigzag graphene nanoribbons for hydrogen storage. A van der Waals-corrected density-functional study. Int. J. Hydrogen Energy 2015, 40, 4960-4968. [CrossRef]

18. Chu, S.; Hu, L.; Hu, X.; Yang, M.; Deng, J. Titanium-embedded graphene as high-capacity hydrogen-storage media. Int. J. Hydrogen Energy 2011, 36, 12324-12328. [CrossRef]

19. Liu, Y.; Ren, L.; He, Y.; Cheng, H.-P. Titanium-decorated graphene for high-capacity hydrogen storage studied by density functional simulations. J. Phys. Condens. Matter 2010, 22, 445301. [CrossRef] [PubMed]

20. Rojas, M.I.; Leiva, E.P.M. Density functional theory study of a graphene sheet modified with titanium in contact with different adsorbates. Phys. Rev. B 2007, 76. [CrossRef]

21. Kim, G.; Jhi, S.-H.; Park, N.; Louie, S.G.; Cohen, M.L. Optimization of metal dispersion in doped graphitic materials for hydrogen storage. Phys. Rev. B 2008, 78. [CrossRef]

22. Luo, Z.; Fan, X.; Pan, R.; An, Y. A first-principles study of Sc-decorated graphene with pyridinic-N defects for hydrogen storage. Int. J. Hydrogen Energy 2017, 42, 3106-3113. [CrossRef]

23. Faye, O.; Szpunar, J.A.; Szpunar, B.; Beye, A.C. Hydrogen adsorption and storage on Palladium—Functionalized graphene with NH-dopant: A first principles calculation. Appl. Surf. Sci. 2017, 392, 362-374. [CrossRef]

24. Faye, O.; Eduok, U.; Szpunar, J.; Szpunar, B.; Samoura, A.; Beye, A. Hydrogen storage on bare Cu atom and Cu-functionalized boron-doped graphene: A first principles study. Int. J. Hydrogen Energy 2017, 42, 4233-4243. [CrossRef]

25. Liu, W.; Liu, Y.; Wang, R. Prediction of hydrogen storage on Y-decorated graphene: A density functional theory study. Appl. Surf. Sci. 2014, 296, 204-208. [CrossRef]

26. Sivek, J.; Sahin, H.; Partoens, B.; Peeters, F.M. Adsorption and absorption of boron, nitrogen, aluminum, and phosphorus on silicene: Stability and electronic and phonon properties. Phys. Rev. B 2013, 87. [CrossRef]

27. Vogt, P.; De Padova, P.; Quaresima, C.; Avila, J.; Frantzeskakis, E.; Asensio, M.C.; Resta, A.; Ealet, B.; Le Lay, G. Silicene: Compelling Experimental Evidence for Graphenelike Two-Dimensional Silicon. Phys. Rev. Lett. 2012, 108. [CrossRef] [PubMed]

28. Zhao, H. Strain and chirality effects on the mechanical and electronic properties of silicene and silicane under uniaxial tension. Phys. Lett. A 2012, 376, 3546-3550. [CrossRef]

29. Liu, H.; Neal, A.T.; Zhu, Z.; Luo, Z.; Xu, X.; Tománek, D.; Ye, P.D. Phosphorene: An Unexplored 2D Semiconductor with a High Hole Mobility. ACS Nano 2014, 8, 4033-4041. [CrossRef] [PubMed] 
30. Zhang, S.; Yan, Z.; Li, Y.; Chen, Z.; Zeng, H. Atomically Thin Arsenene and Antimonene: Semimetal-Semiconductor and Indirect-Direct Band-Gap Transitions. Angew. Chem. Int. Ed. 2015, 54, 3112-3115. [CrossRef] [PubMed]

31. Xie, X.; Zhou, Y.; Bi, H.; Yin, K.; Wan, S.; Sun, L. Large-range Control of the Microstructures and Properties of Three-dimensional Porous Graphene. Sci. Rep. 2013, 3. [CrossRef] [PubMed]

32. Bieri, M.; Treier, M.; Cai, J.; Aït-Mansour, K.; Ruffieux, P.; Gröning, O.; Gröning, P.; Kastler, M.; Rieger, R.; Feng, X.; et al. Porous graphenes: Two-dimensional polymer synthesis with atomic precision. Chem. Commun. 2009. [CrossRef] [PubMed]

33. Huang, C.; Wu, H.; Deng, K.; Tang, W.; Kan, E. Improved permeability and selectivity in porous graphene for hydrogen purification. Phys. Chem. Chem. Phys. 2014, 16, 25755-25759. [CrossRef] [PubMed]

34. Reunchan, P.; Jhi, S.-H. Metal-dispersed porous graphene for hydrogen storage. Appl. Phys. Lett. 2011, 98, 93103. [CrossRef]

35. Yuan, L.; Chen, Y.; Kang, L.; Zhang, C.; Wang, D.; Wang, C.; Zhang, M.; Wu, X. First-principles investigation of hydrogen storage capacity of Y-decorated porous graphene. Appl. Surf. Sci. 2017, 399, 463-468. [CrossRef]

36. Sun, J.; Wang, H.-T.; He, J.; Tian, Y. Ab initio investigations of optical properties of the high-pressure phases of ZnO. Phys. Rev. B 2005, 71. [CrossRef]

37. Rao, D.; Lu, R.; Meng, Z.; Wang, Y.; Lu, Z.; Liu, Y.; Chen, X.; Kan, E.; Xiao, C.; Deng, K.; et al. Electronic properties and hydrogen storage application of designed porous nanotubes from a polyphenylene network. Int. J. Hydrogen Energy 2014, 39, 18966-18975. [CrossRef]

38. Brunetto, G.; Autreto, P.A.S.; Machado, L.D.; Santos, B.I.; Dos Santos, R.P.B.; Galvão, D.S. Nonzero Gap Two-Dimensional Carbon Allotrope from Porous Graphene. J. Phys. Chem. C 2012, 116, 12810-12813. [CrossRef]

39. Li, Y.; Zhou, Z.; Shen, P.; Chen, Z. Two-dimensional polyphenylene: Experimentally available porous graphene as a hydrogen purification membrane. Chem. Commun. 2010, 46, 3672. [CrossRef] [PubMed]

40. Ao, Z.M.; Peeters, F.M. High-capacity hydrogen storage in Al-adsorbed graphene. Phys. Rev. B 2010, 81. [CrossRef]

41. Bhattacharya, A.; Bhattacharya, S.; Majumder, C.; Das, G.P. Transition-Metal Decoration Enhanced Room-Temperature Hydrogen Storage in a Defect-Modulated Graphene Sheet. J. Phys. Chem. C 2010, 114, 10297-10301. [CrossRef]

42. Ataca, C.; Aktürk, E.; Ciraci, S. Hydrogen storage of calcium atoms adsorbed on graphene: First-principles plane wave calculations. Phys. Rev. B 2009, 79. [CrossRef]

43. Chen, M.; Yang, X.-B.; Cui, J.; Tang, J.-J.; Gan, L.-Y.; Zhu, M.; Zhao, Y.-J. Stability of transition metals on $\mathrm{Mg}(0001)$ surfaces and their effects on hydrogen adsorption. Int. J. Hydrogen Energy 2012, 37, 309-317. [CrossRef]

(C) 2017 by the authors. Licensee MDPI, Basel, Switzerland. This article is an open access article distributed under the terms and conditions of the Creative Commons Attribution (CC BY) license (http://creativecommons.org/licenses/by/4.0/). 